\title{
An Analogue of Holstein-Primakoff and Dyson Realizations for Lie Superalgebras. The Lie Superalgebra $s l(1 / n)$
}

\author{
T.D. PALEV \\ International Centre for Theoretical Physics, P.O. Box 586, 34100 Trieste, Italy \\ and \\ International School for Advanced Studies, via Beirut 2-4, 34123 Trieste, Italy \\ Permanent address: \\ Institute for Nuclear Research and Nuclear Energy, 1784 Sofia, Bulgaria; \\ E-mail: tpalev@inrne.acad.bg
}

\begin{abstract}
An analogue of the Holstein-Primakoff and Dyson realizations for the Lie superalgebra $s l(1 / n)$ is written down. Expressions are the same as for the Lie algebra $\operatorname{sl}(n+1)$, however in the latter, Bose operators have to be replaced with Fermi operators.
\end{abstract}

In the present letter, we extend the concept of Holstein-Primakoff (H-P) and Dyson (D) realizations to the case of Lie superalgebras and, more precisely, to the Lie superalgebras $\operatorname{sl}(1 / n)$ for any $n$.

Initially, the H-P and D realizations were given for $\operatorname{sl}(2)[1,2]$. It took some time for the results to be generalized to the Lie algebras $g l(n)[3], s o(2 n)[4]$ and $s p(2 n)$ [5]. The extension to the case of quantum algebras is available so far only for $s l(2)[6]$ and $s l(3)$ [7]. To our best knowledge, results on $\mathrm{H}-\mathrm{P}$ or $\mathrm{D}$ realizations for Lie superalgebras were not published in the literature so far.

The motivation for the present work stems from the observation that Holstein-Primakoff and Dyson realizations play important role in various branches of physics. It is not possible to mention all contributions. For applications in nuclear physics, see $[8,9]$ and references therein; some early applications in the spin-wave theory can be found in [10] (see [11] for more recent results), but there are, certainly, several other publications.

The H-P and D realization for $\operatorname{sl}(1 / n)$ we are going to present is an analogue of the one given by Okubo [3] for the Lie algebra $s l(n)$. Nevertheless, it is only an analogue. The point is that we give a realization of the generators in terms of Fermi creation and annihilation operators (CAOs), whereas all H-P and D realizations are imbeddings into enveloping algebras of Bose CAOs. The similarity, however, is striking: replacing in the expressions for $s l(n+1)$ Bose CAOs, with Fermi CAOs one obtains the H-P and the D realizations for $s l(1 / n)$. 
We proceed to recall the definition of the Lie superalgebra (LS) $s l(1 / n)$ in the notation we are going to use. Let $U$ be the (free complex) associative unital (= with unity) algebra of indeterminants $a_{1}^{ \pm}, \ldots, a_{n}^{ \pm}$subject to the relations (below and throughout $[x, y]=x y-y x$, $\{x, y\}=x y+y x)$

$$
\begin{aligned}
& {\left[\left\{a_{i}^{+}, a_{j}^{-}\right\}, a_{k}^{+}\right]=\delta_{j k} a_{i}^{+}-\delta_{i j} a_{k}^{+}, \quad\left[\left\{a_{i}^{+}, a_{j}^{-}\right\}, a_{k}^{-}\right]=-\delta_{i k} a_{j}^{-}+\delta_{i j} a_{k}^{-},} \\
& \left\{a_{i}^{+}, a_{j}^{+}\right\}=\left\{a_{i}^{-}, a_{j}^{-}\right\}=0 .
\end{aligned}
$$

For the sake of convenience, we refer to $a_{i}^{+}$and to $a_{i}^{-}$as to creation and annihilation operators, respectively. Introduce a $\mathbb{Z}_{2}$-grading in $U$, setting

$$
\operatorname{deg}\left(a_{i}^{ \pm}\right)=\overline{1}, \quad i=1, \ldots, n .
$$

Then $U$ is an (infinite-dimensional) associative superalgebra, which is also a LS with respect to the product (supercommutator) defined between every two homogeneous elements $x, y \in U$ as

$$
\llbracket x, y \rrbracket=x y-(-1)^{\operatorname{deg}(x) \operatorname{deg}(y)} y x ;
$$

its finite-dimensional subspace

$$
A=\text { lin.env. }\left\{a_{i}^{ \pm},\left\{a_{j}^{+}, a_{k}^{-}\right\} \mid i, j, k=1, \ldots, n\right\} \subset U
$$

is a Lie superalgebra with odd generators $a_{1}^{ \pm}, \ldots, a_{n}^{ \pm}$.

Proposition 1 The LS $A$ is (isomorphic to) $s l(1 / n), A=s l(1 / n) . U$ is its universal enveloping algebra, $U=U[\operatorname{sl}(1 / n)]$.

Proof. The proof can be found in [12]. We recall the main steps. Let $e_{A, B}, A, B=$ $0,1,2, \ldots, n$, be an $(n+1) \times(n+1)$ matrix with 1 on the intersection of the $A^{\text {th }}$ row and $B^{t h}$ column and zero elsewhere. Then (in a lowest exact matrix representation [13]) $s l(1 / n)$ is a direct space sum of its even subalgebra

$$
\operatorname{sl}(1 / n)_{\overline{0}}=g l(n)=\text { lin.env. }\left\{e_{i j}+\delta_{i j} e_{00} \mid i, j=1, \ldots, n\right\}
$$

and the odd subspace

$$
\operatorname{sl}(1 / n)_{\overline{1}}=\text { lin.env. }\left\{e_{0 i}, e_{i 0} \mid i=1, \ldots, n\right\} .
$$

The one-to-one correspondence between the representation-independent Cartan-Weyl generators (4) and the generators in the above representation reads:

$$
a_{i}^{+} \leftrightarrow e_{i 0}, \quad a_{i}^{-} \leftrightarrow e_{0 i}, \quad\left\{a_{i}^{+}, a_{j}^{-}\right\} \leftrightarrow e_{i j}+\delta_{i j} e_{00}, \quad i, j=1, \ldots, n .
$$

It is straightforward to verify that the above map is an LS isomorphism. In particular only from relations (1) (and the graded Jacoby identity), one derives also the commutation relations between all even generators

$$
\left[\left\{a_{i}^{+}, a_{j}^{-}\right\},\left\{a_{k}^{+}, a_{l}^{-}\right\}\right]=\delta_{j k}\left\{a_{i}^{+}, a_{l}^{-}\right\}-\delta_{i l}\left\{a_{k}^{+}, a_{j}^{-}\right\} .
$$

By definition, $U[\operatorname{sl}(1 / n)]$ is the associative unital algebra of $a_{1}^{ \pm}, \ldots, a_{n}^{ \pm}$subject to relations (1) and (8). Since (8) follow from (1), one can skip them. Therefore, $U=U[s l(1 / n)]$. 
Let $f_{i}^{ \pm}, i=1, \ldots, n$ be $n$ pairs of Fermi CAOs,

$$
\left\{f_{i}^{+}, f_{j}^{-}\right\}=\delta_{i j}, \quad\left\{f_{i}^{+}, f_{j}^{+}\right\}=\left\{f_{i}^{-}, f_{j}^{-}\right\}=0 .
$$

Denote by $W(0 / n)$ the Fermi superalgebra, namely the $4^{n}$-dimensional associative unital algebra with relations $(9)$ and $\mathbb{Z}_{2}$ - grading induced from $\operatorname{deg}\left(f_{i}^{ \pm}\right)=\overline{1}$.

Consider the following operators in $W(0 / n)$

$$
\varphi\left(a_{i}^{+}\right)=f_{i}^{+}, \quad \varphi\left(a_{i}^{-}\right)=(p-N) f_{i}^{-}, \quad \text { where } N=\sum_{k=1}^{n} f_{k}^{+} f_{k}^{-}, \quad i=1, \ldots, n .
$$

It is an easy exercise to verify that operators (10) satisfy relations (1) and, moreover, they are linearly independent. Thus, we have obtained the following result.

Proposition 2 The map $\varphi$ defines an isomorphism of $s l(1 / n)$ into $W(0 / n)$ for any complex number $p$.

This realization of $\operatorname{sl}(1 / n)$ in $W(n)$ is an analogue of the Dyson realization of $s l(n+1)$ [2]. The latter can be formally obtained from (10) replacing Fermi operators with Bose CAOs

$$
\left[b_{i}^{-}, b_{j}^{+}\right]=\delta_{i j}, \quad\left[b_{i}^{+}, b_{j}^{+}\right]=\left[b_{i}^{-}, b_{j}^{-}\right]=0 .
$$

In that case, the linear envelope of

$$
b_{i}^{+}, \quad(p-N) b_{i}^{-}, \quad \text { and }\left[b_{i}^{+},(p-N) b_{j}^{-}\right], \quad i, j=1, \ldots, n
$$

spans a realization of $g l(n+1)$.

The Dyson realization defines a $2^{n}$-dimensional representation of $s l(1 / n)$ in the Fermi Fock space $F(0 / n)$. Let

$$
|p ; \Theta\rangle \equiv\left|p ; \theta_{1}, \theta_{2}, \ldots, \theta_{n}\right\rangle=\left(f_{1}^{+}\right)^{\theta_{1}}\left(f_{2}^{+}\right)^{\theta_{2}} \ldots\left(f_{n}^{+}\right)^{\theta_{n}}|0\rangle, \quad \theta_{1}, \ldots, \theta_{n}=0,1,
$$

be a usual orthonormed Fock basis in $F(0 / n)$ and let $|p ; \Theta\rangle_{ \pm i}$ be a vector obtained from $|p ; \Theta\rangle$ after the replacement of $\theta_{i}$ with $\theta_{i} \pm 1$. The transformations of the basis under the action of the $s l(1 / n)$ generators read:

$$
\begin{aligned}
& \varphi\left(a_{i}^{+}\right)|p ; \Theta\rangle=\left(1-\theta_{i}\right)(-1)^{\theta_{1}+\ldots+\theta_{i-1}}|p ; \Theta\rangle_{+i}, \\
& \varphi\left(a_{i}^{-}\right)|p ; \Theta\rangle=\theta_{i}(-1)^{\theta_{1}+\ldots+\theta_{i-1}}\left(p-\sum_{k=1}^{n} \theta_{k}+1\right)|p ; \Theta\rangle_{-i} .
\end{aligned}
$$

In the generic case, the representation of $s l(1 / n)$ in $F(0 / n)$ is irreducible. If, however, $p=1,2,3, \ldots, n-1$, the representation is indecomposible. Due to the factor $\left(p-\sum_{k=1}^{n} \theta_{k}+\right.$ $1)$, the subspace of all vectors with $\sum_{k=1}^{n} \theta_{k}>p$ is an invariant subspace $F(0 / n)_{i n v} \subset$ $F(0 / n)$. The factor space $F(0 / n)_{i r r}=F(0 / n) / F(0 / n)_{i n v}$ is irreducible. By definition, such representations are called atypical [13].

The advantage of the Dyson realization (10) is its simplicity. Its disadvantage stems from the observation that the representation is not unitary, i.e., the Hermitian conjugate to $\varphi\left(a_{i}^{-}\right)$is not equal to $\varphi\left(a_{i}^{+}\right)$, i.e.,

$$
\left(\varphi\left(a_{i}^{-}\right)\right)^{\dagger}=\varphi\left(a_{i}^{+}\right)
$$


does not hold.

It turns out all representations in $F(0 / n)_{i r r}$ corresponding to nonnegative integers $p, p \in \mathbf{Z}_{+}$, are equivalent to unitary representations. To this end, one has to change the scalar product in $F(0 / n)_{i r r}$ in an appropriate way. We give the final results.

Proposition 3 The map $\pi$ defined as

$$
\pi\left(a_{i}^{+}\right)=f_{i}^{+} \sqrt{p-N}, \quad \pi\left(a_{i}^{-}\right)=\sqrt{p-N} f_{i}^{-}
$$

is an isomorphism of $\operatorname{sl}(1 / n)$ into $W(0 / n)$ for any $p \in \mathbf{Z}_{+}$. The space $F(0 / n)_{i r r}$ with an orthonormed basis consisting of all vectors

$$
|p ; \Theta\rangle \equiv\left|p ; \theta_{1}, \theta_{2}, \ldots, \theta_{n}\right\rangle, \quad \text { such that } \sum_{k=1}^{n} \theta_{k} \leq p, \quad \theta_{1}, \ldots, \theta_{n}=0,1,
$$

carries an unitary irreducible representation of $s l(1 / n)$, i.e., (15) holds. The transformation of the basis reads:

$$
\begin{aligned}
& \pi\left(a_{i}^{+}\right)|p ; \Theta\rangle=\left(1-\theta_{i}\right)(-1)^{\theta_{1}+\ldots+\theta_{i-1} \sqrt{p-\sum_{k=1}^{n} \theta_{k}}}|p ; \Theta\rangle_{+i}, \\
& \pi\left(a_{i}^{-}\right)|p ; \Theta\rangle=\theta_{i}(-1)^{\theta_{1}+\ldots+\theta_{i-1}} \sqrt{p-\sum_{k=1}^{n} \theta_{k}+1}|p ; \Theta\rangle_{-i} .
\end{aligned}
$$

If $p<n$, the representation is atypical.

The supercommutation relations (1) can be checked within the particular representation, acting on the basis vectors (17) according to (18). One can proceed in a more algebraic way first expanding $\sqrt{p-N}$ into a series with respect to $N$, so that it is explicit that $\sqrt{p-N} \in W(0 / n)$.

Expressions (16) can be considered as an analogue of the Holstein-Primakoff realization for the Lie superalgebra $s l(1 / n)$. Also here, replacing fermions in (16) with bosons, one obtains the H-P realizations for $\mathrm{sl}(\mathrm{n}+1)$ as given by Okubo [3].

We wrote down explicit expressions in terms of fermions only $\pi\left(a_{i}^{ \pm}\right)$. The rest of the Cartan-Weyl generators, namely $\pi\left(\left\{a_{i}^{+}, a_{j}^{-}\right\}\right)=\left\{\pi\left(a_{i}^{+}\right), \pi\left(a_{j}^{-}\right)\right\}$, follows from (16). One can express also the unit operator in $F(0 / n)_{i r r}$ in terms of fermions, thus extending the results to the $\mathrm{LS} g l(1 / \mathrm{n})$. The more detailed results will be given elsewhere directly for the $\operatorname{LS} \mathrm{gl}(\mathrm{m} / \mathrm{n})$.

Let us note that the explicit expressions for all finite-dimensional irreducible representations of $s l(1 / n)$ and $g l(1 / n)$ are known [14]. The formulas are however extremely involved. Here we have obtained a small subset of all representations, which description is, however, simple and is realized in Fock spaces familiar for physics.

The above construction holds also for the case $n=\infty$. In that case, the representation space of $s l(1 / \infty)$ has an orthonormed basis consisting of all vectors such that $\sum_{k=1}^{\infty} \theta_{k} \leq p$. All representations are atypical infinite-dimensional irreducible representations.

One could expect that the above results may help to give H-P and D realizations for the quantum algebra $U_{q}[s l(1 / n)]$. The explicit representations of the Chevalley generators in a Gelf́and-Zetlin basis are known [15]. As a first step, here one has to pass to the corresponding deformed CAOs, expressing them in terms of the Chevalley operators, and write subsequently the transformation of the basis under the action of these operators. 


\section{Acknowledgments}

The author is thankful to Prof. Randjbar-Daemi for the the kind hospitality at the High Energy Section of ICTP, where most of the results were obtained. He is grateful to Prof. C. Reina for making it possible to visit the Section on Mathematical physics in Sissa, where the paper was completed. The work was supported by the Grant $\Phi-416$ of the Bulgarian Foundation for Scientific Research.

\section{References}

[1] Holstein T. and Primakoff H., Field dependence of the intrinsic domain magnetization of a ferromagnet, Phys. Rev., 1940, V.58, 1098-1113.

[2] Dyson F.J., General theory of spin-wave interactions, Phys. Rev., 1956, V.102, 1217-1230.

[3] Okubo S., Algebraic identities among $U(n)$ infinitesimal generators, J. Math. Phys., 1975, V.16, $528-536$.

[4] Papanicolaou N., Pseudo-spin and classical correspondence for Fermi fields, Ann. Phys. (NY), 1981, V.136, 210-225.

[5] Deenen J. and Quesne C., Dynamical group of microscopic collective systems. I. One-dimensional case, J. Math. Phys., 1982, V.23, 878-889.

Deenen J. and Quesne C., Dynamical group of microscopic collective systems. II. Boson representaions in $d$ dimensions, J. Math. Phys., 1982, V.23, 2004-2015.

[6] Chaichian M., Ellinas D. and Kulish P.P., Quantum algebra as the dynamical symmetry of the deformed Jaynes-Cummings model, Phys. Rev. Lett., 1990, V.65, 980-983.

Quesne C., Coherent states, $K$-matrix theory and $q$-boson realization of the quantum algebra $s u_{q}(2)$, Phys. Lett. A, 1991, V.153, 303-307.

Chakrabarti R. and Jagannathan R., A $(p, q)$-oscillator realization of two-parameter quantum algebras, J. Phys. A, 1991, V.24, L711-L718.

Katriel J. and Solomon A.I., Generalized $q$-bosons and their squeezed states, J. Phys. A, 1991, V.24, 2093-2105.

Yu Z.R., Some realizations of the quantum algebra $U_{q}(s u(2))$, J. Phys. A, 1991, V.24, L1321-L1325. Kundu A. and Basu Mallich B., q-Deformation of the Holstein-Primakoff transformation and other bosonizations of the quantum group, Phys. Lett. A, 1991, V.156, 175-178.

Pan F., A new realization of the quantum group $S U(2)_{q}$, Chin. Phys. Lett., 1991, V.8, 56-58.

[7] da-Providencia J., Mean-field generation of the classical q-deformation of su(3), J. Phys. A, 1993, V.26, 5845-5849.

[8] Klein A. and Marshalek E.R., Boson realization of Lie algebras with applications to nuclear physics, Rev. Mod. Phys., 1991, V.63, 375-558.

[9] Ring P. and Schuck P., The Nuclear Many-Body Problem, Springer-Verlag, New York, Heidelberg, Berlin.

[10] Kittel C., Quantum Theory of Solids, Willey, New York, 1963.

[11] Caspers W.J., Spin Systems, World Sci. Pub. Co., Inc., Teanek, NJ, 1989.

[12] Palev T.D., Fock space representations of the Lie superalgebra A(0,n), J. Math. Phys., 1982, V.21, 1293-1298.

[13] Kac V.G., Representations of classical Lie superalgebras, Lect. Notes Math., 1978, V.676, 597-626. 
[14] Palev T.D., Finite-dimensional representations of the special linear Lie superalgebra sl(1/n). I.Typical representations, J. Math. Phys., 1987, V.28, 2280-2303;

Finite-dimensional representations of the special linear Lie superalgebra $s l(1, n)$. II. Nontypical representations, J. Math. Phys., 1988, V.29, 2589-2598;

Irreducible finite-dimensional representations of the Lie superalgebra $g l(n / 1)$ in a Gel'fand-Zetlin basis, J. Math. Phys., 1989, V.30, 1433-1442.

Palev T. D., Irreducible finite-dimensional representations of the Lie superalgebras $g l(n / 1)$ in a Gel'fand-Zetlin basis, Funkt. Anal. Prilozh., 1987, V.21, \#3, 85-86 (In Russian); Funct. Anal. Appl., 1987, V.21, 245-246 (English transl.).

[15] Palev T.D. and Tolstoy V.N., Finite-dimensional irreducible representations of the quantum superalgebra $U_{q}[g l(n / 1)]$, Comm. Math. Phys., 1991, V.141, 549-556. 